\title{
Analysis and improvement of ramp gain error in single-ramp single-slope ADCs for CMOS image sensors
}

\author{
Xu Cheng ${ }^{\mathrm{a}, *}$, Xiaoyang Zeng ${ }^{\mathrm{a}}$, Qi Feng ${ }^{\mathrm{b}}$ \\ ${ }^{a}$ State Key Laboratory of ASIC and System, Fudan University, Shanghai, China \\ ${ }^{b}$ Key Laboratory of Infrared Imaging Materials and Detectors, Shanghai Institute of \\ Technical Physics, Chinese Academy of Sciences, Shanghai, China
}

\begin{abstract}
The single-ramp single-slope (SRSS) analog-to-digital converter (ADC) is a promising candidate for column-parallel architectures. This paper, for the first time, quantitatively analyzes the ramp gain error in the ramp-input stage (RIS) that is the crucial component in such ADC, and verifies it using behavior model simulation. In contrast to the conventional passive RIS, an active RIS is proposed to alleviate the ramp gain error. With an active feedback loop, the ramp gain becomes a product of two reciprocal ratios of capacitance, whose departure from unity is attributed to the relative deviations rather than the absolute values in the passive RIS. The proposed concept is designed and verified by simulations in a $0.18-\mu m$ CMOS process.

Keywords: CMOS image sensor (CIS), analog-to-digital converter (ADC), column-parallel ADC, single-slope ADC, two-step ADC
\end{abstract}

\section{Introduction}

A CMOS image sensor (CIS) provides the significant advantages over charge coupled devices (CCDs) due to lower power consumption and easier System on Chip (SoC) integration. A column-parallel architecture has become popular for 5 analog-to-digital converter (ADC) integration in CIS, in that it features a good trade-off among frame rate, column number, noise, and power consumption.

*chengxu@fudan.edu.cn 


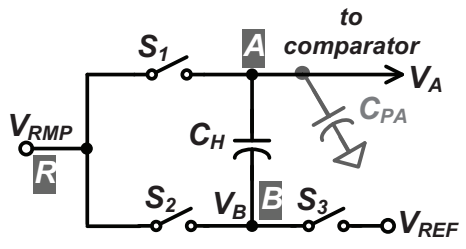

(a)

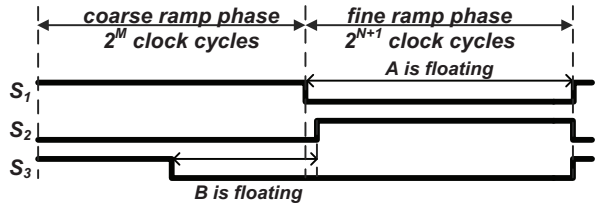

(b)

Figure 1: Conventional passive ramp input stage: (a) schematic with $V_{R M P}$ from a ramp generator and $V_{A}$ to a comparator, and (b) timing sequence.

There are many types of ADCs employed for column-parallel architectures, such as single-slope (SS) ADCs [1 4], successive approximation register (SAR) ADCs [5] 9], $\Delta-\Sigma$ ADCs [10], and cyclic ADCs [11, 12].

Among them, the SS ADC is most widely used for its good uniformity and circuit simplicity, although it requires $2^{B}$ clock cycles for $B$ bit conversion. To speed up the conversion, the multiple-ramp SS (MRSS) ADCs [13] divides $B$-bit conversion into $M$-bit coarse and $N$-bit fine conversions, so as to shorten the conversion cycles to $\left(2^{M}+2^{N}\right)$. And the final output code is obtained as

$$
D=2^{N} \cdot m+n
$$

10 spectively. Nevertheless, the required $2^{M}$ fine ramp generators not only occupy large area, but more seriously suffer from the mismatch of the ramp slopes and offsets, both of which limit the coarse ramp resolution $M$ to only 2 or 3 bits with little speed improvement. For faster conversion, the single-ramp SS (SRSS)

15 ADCs [14] memorize the coarse ramp voltage individually by using a holding capacitor $\left(C_{H}\right)$ and two switches as shown in Fig. [1](a), termed passive ramp input stage (RIS) in this paper. Hence, only two ramp generators are required, and the conversion cycles become $\left(2^{M}+2^{N+1}\right)$ due to offset voltage correction.

However, the passive RIS suffers from parasitic capacitance during the fine ramp phase. As shown in Fig. 1, the fine ramp is transferred from $R$ to $A$ via $B$ and $C_{H}$, whose top plate $A$ is floating and thus sensitive to charge injection, 
clock feedthrough, and parasitic capacitance. The former two induce an offset voltage $\left(\Delta_{O S}\right)$, which has been made signal-independent and covered by the fine ramp extension [14]. The parasitic capacitance $\left(C_{P A}\right.$ in gray in Fig. [1](a)] causes the fine ramp gain $(G)$ deviated from unity given by

$$
G=\frac{\Delta V_{A}}{\Delta V_{R}}=\frac{\Delta V_{A}}{\Delta V_{B}}=\frac{C_{H}}{C_{H}+C_{P A}}<1,
$$

leading to overall nonlinearity unless $C_{H}$ is large enough, which makes it difficult

20 to optimize the conversion speed for high resolutions. This problem has been neither analyzed, nor solved by far. An alternative approach proposed in [15] avoids this problem by replacing $C_{H}$ with $2^{M} \mathrm{DAC}$ (digital-to-analog converter) switches controlled by the latched $m$. However, two separate DAC-based ramp generators require the same resolution, and the local hardware expense in each column may also limit $M$.

Therefore, this paper firstly quantifies the ramp gain error effect on linearity of the SRSS ADCs, termed linearity constraint, and verifies it by behavior model simulation. In order to improve linearity, an active RIS is proposed and verified by simulation. The conclusions are drawn at the end of this paper.

\section{зо 2. Linearity constraint}

The following analysis is based on the SRSS ADC with the fine ramp expanding by $\pm 0.5 \Delta_{C}$ or $\pm 2^{N-1} \Delta_{F}$ [14], where $\Delta_{C}=\frac{V_{F S}}{2^{M}}, \Delta_{F}=L S B=\frac{V_{F S}}{2^{M+N}}$, and $V_{F S}$ is the full scale voltage. As shown in Fig. 2, the $m^{\text {th }}$ fine ramp under investigation consists of three parts termed negative extension, main fine ramp,

35 and positive extension, where a horizontal line and the following vertical line are termed the code and the corresponding code width, respectively. Although only $G<1$ arises in the passive RIS in Fig. 1(a), both $G<1$ and $G>1$ will be examined in that both cases do arise in the proposed RIS.

\subsection{Basic properties}

40 Two properties can be easily obtained in the RIS operation. Firstly, a code in the $m^{\text {th }}$ fine ramp does not appear in the final output codes unless its step partly 


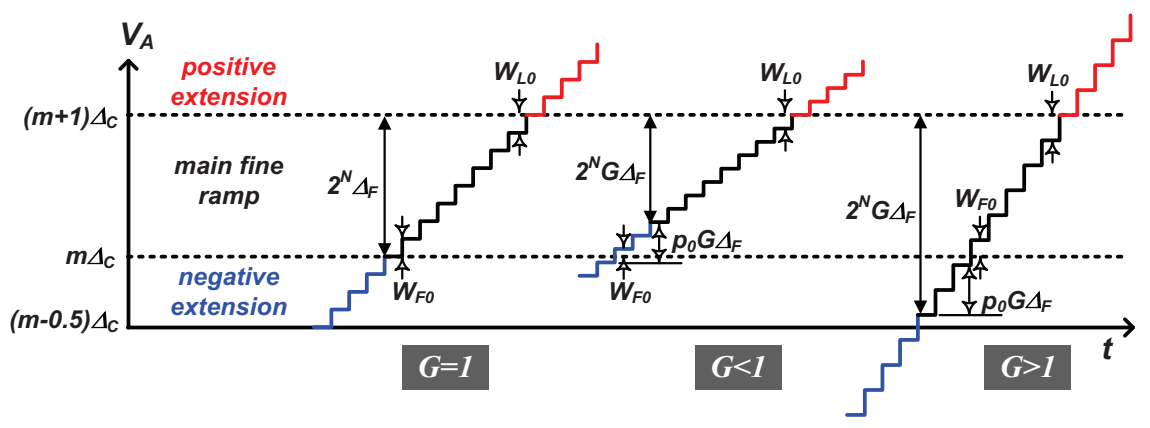

Figure 2: The $m^{\text {th }}$ 3-bit fine ramp with different fine ramp gain $(G)$ and without offset voltage $\left(\Delta_{O S}=0\right)$.

or totally falls into $\left[m \Delta_{C},(m+1) \Delta_{C}\right)$ which is set by the coarse ramp. Secondly, if there is no offset voltage, i.e., $\Delta_{O S}=0$, the $m^{\text {th }}$ positive extension always starts with $(m+1) \Delta_{C}$ regardless of $G$. This can be explained as follows. At the end of the coarse ramp phase, $(m+1) \Delta_{C}$ is stored on Node $\mathrm{A}$ as $V_{A}$. Then, Node B is switched from $V_{R E F}$ to $V_{R M P}$, making $V_{A}$ decreased by $\left(2^{N}+2^{N-1}\right) G$ in LSB. In the fine ramp phase, $V_{A}$ is increased by $G$ every step and reaches $(m+1) \Delta_{C}$ after completing the negative extension and the main fine ramp that contain $\left(2^{N-1}+2^{N}\right)$ number of steps in total.

For the convenience of discussion, the final output codes in the same $\left(m^{t h}\right)$ fine ramp are categorized into four types according to the step locations of their fine ramp codes with respect to $\left[m \Delta_{C},(m+1) \Delta_{C}\right)$.

1) The totally appearing code (TAC) is defined as a final output code with its fine step completely inside $\left[m \Delta_{C},(m+1) \Delta_{C}\right)$, and its code width always equals $G$.

2) The first appearing code (FAC) is defined as a final output code with its fine step across $m \Delta_{C}$, above which the step portion is its code width denoted as $W_{F 0}$ and $W_{F}$ for $\Delta_{O S}=0$, and $\Delta_{O S} \neq 0$, respectively.

3) The last appearing code (LAC) is defined as a final output code with its fine step across $(m+1) \Delta_{C}$, below which the step portion is its code width denoted as $W_{L 0}$ and $W_{L}$ for $\Delta_{O S}=0$, and $\Delta_{O S} \neq 0$, respectively. 
Obviously, $W_{L 0}=G$ based on the second property.

4) The non-linear code (NLC) is either missing code or a pair of duplicate codes, the number of which in each fine ramp is denoted as $p_{0}$ and $p$ for $\Delta_{O S}=0$, and $\Delta_{O S} \neq 0$, respectively. Since (10) can be rewritten as $D=2^{N} \cdot m+n=2^{N} \cdot(m-1)+\left(n+2^{N}\right)$, the same final output code is possibly made up of different pairs of coarse and fine codes, termed a pair of duplicate codes. When NLC is a pair of duplicate codes, its code width is the sum of both code widths, and the number of NLCs is defined positive. When NLC is missing codes, its code width is 0 , and the number of NLCs is defined negative.

Based on the two properties aforementioned, the widths of FAC and LAC as well as the number of NLC will be examined in details in Section 2.2 and 2.3 .

\section{2. $G \neq 1$ and $\Delta_{O S}=0$}

In this case as shown in Fig. 2. $W_{L 0}=G$ according to the second property, but $W_{F 0}$ varies depending on $G$ explained as below. When $G<1$, the $m^{\text {th }}$ fine ramp is squeezed towards $(m+1) \Delta_{C}$. Once their steps have been partly or totally squeezed above $m \Delta_{C}, p_{0}$ codes in the negative extension, that used to be invisible, appear and become duplicate with the codes in the $(m-1)^{t h}$ main fine ramp. Therefore, $W_{F 0}$ becomes

$$
W_{F 0}=2^{N}(1-G)-\left(p_{0}-1\right) G
$$

in unit of $\Delta_{F}$, where $p_{0}$ is an integer making $0<W_{F 0} \leqslant G$, and consequently satisfies

$$
p_{0}=\left\lceil 2^{N}\left(\frac{1}{G}-1\right)\right\rceil .
$$

75 When $G>1$ in Fig. 2, the $m^{t h}$ fine ramp is stretched away from $(m+1) \Delta_{C}$. Once their steps have been totally stretched below $m \Delta_{C}, p_{0}$ codes in the main fine ramp, that used to be visible, disappear and become missing codes. Because the number of missing codes is negative, (3) also applies to $G>1$.

Using (3), a ramp gain range can be derived as follows to guarantee linearity, termed linearity constraint. Monotonicity can be interpreted as a lower bound 
Table 1: Code widths and non-linear code numbers with offset voltage.

\begin{tabular}{c|c|c|c|c}
\hline Case & $r=\Delta_{O S} \% G$ & $W_{F}$ & $W_{L}$ & $p$ \\
\hline 1 & $G-W_{F 0}<r<G$ & $W_{F 0}+r-G$ & $W_{L 0}-r$ & $p_{0}+1$ \\
\hline 2 & $0 \leqslant r \leqslant G-W_{F 0}$ & $W_{F 0}+r$ & $W_{L 0}-r$ & $p_{0}$ \\
\hline 3 & $-W_{F 0}<r<0$ & $W_{F 0}+r$ & $-r$ & $p_{0}+1$ \\
\hline 4 & $-G<r \leqslant-W_{F 0}$ & $W_{F 0}+G+r$ & $-r$ & $p_{0}$ \\
\hline
\end{tabular}

of $G$ so that only the greatest code in the negative extension can have its step partly squeezed above $m \Delta_{C}$, which is equivalent to $p_{0}=1$ and $W_{F 0}<G$. Meanwhile, no missing codes can be interpreted as an upper bound of $G$ so that only the smallest code in the main fine ramp can have its step partly stretched below $m \Delta_{C}$, which is equivalent to $p_{0}=0$ and $W_{F 0}>0$. Substituting these two conditions into (3), the linearity constraint is obtained as

$$
1-\frac{1}{2^{N}+1}<G<1+\frac{1}{2^{N}-1} .
$$

\section{3. $G \neq 1$ and $\Delta_{O S} \neq 0$}

A non-zero offset voltage $\Delta_{O S}$ makes the whole fine ramp move vertically, but can be covered if

$$
-2^{N-1} G<\Delta_{O S}<\left(2^{N}+2^{N-1}\right) G-2^{N} .
$$

so Within this range, $\Delta_{O S}$ can be decomposed as $q G+r$ in LSB, where $q$ and $r$ are the quotient and remainder, respectively. $r$ has the same sign as $\Delta_{O S}$, and reflects the $\Delta_{O S}$ effect on non-linearity as explained as follows.

- When $r \geqslant 0$, the whole fine ramp is moved upwards by $r$. As $r$ increases from 0 to $G-W_{F 0}$, both FAC and LAC remain unchanged, and therefore, $p=p_{0}$ with $W_{F}=W_{F 0}+r$ and $W_{L}=W_{L 0}-r$.

- Once $r>G-W_{F 0}$, the FAC becomes a TAC in that its step is totally moved into $\left[m \Delta_{C},(m+1) \Delta_{C}\right)$. Meanwhile, the code just below the old FAC, that used to be invisible, becomes the new FAC with $W_{F}=r-$ 


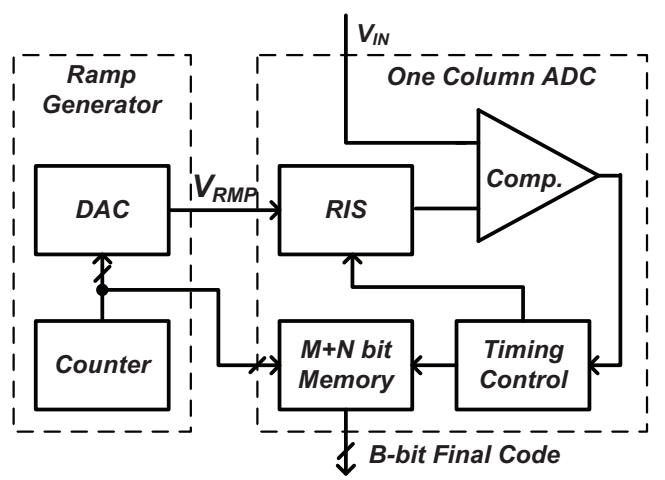

Figure 3: Block diagram of an SRSS ADC.

$\left(G-W_{F 0}\right)$. As a result, the numbers of the duplicate or missing codes are increased and decreased by 1 , respectively, leading to $p=p_{0}+1$. Note that due to $p_{0}<0$ for missing codes, $p_{0}+1$ means that the number of missing codes is decreased by 1 .

- When $r<0$, the whole fine ramp is moved downwards by $|r|$. The LAC becomes a TAC in that its step is totally moved into $\left[m \Delta_{C},(m+1) \Delta_{C}\right)$. Meanwhile, the code just above the old LAC, that used to be invisible, becomes the new LAC with $W_{L}=|r|$. As $r$ decreases from 0 to $\left(-W_{F 0}\right)$, the FAC remains unchanged with $W_{F}=W_{F 0}-|r|$. As a result, $p=p_{0}+1$ due to appearance of one new code.

- Once $r \leqslant-W_{F 0}$, the old FAC disappears due to its step totally moved out of $\left[m \Delta_{C},(m+1) \Delta_{C}\right)$. Meanwhile, the code just above the old FAC, that used to be a TAC, becomes the new FAC with $W_{F}=G-\left(|r|-W_{F 0}\right)$. As a result, $p$ returns to $p_{0}$ due to disappearance of one code.

These four cases are summarized in Table 1 .

\subsection{Verification by behavior model}

In order to support the theory, the SRSS ADC is modeled in the behavior level with adjustable $G$ and $\Delta_{O S}$. This behavior model describes the static 


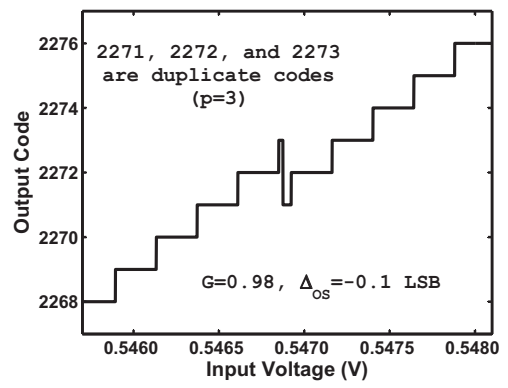

(a)

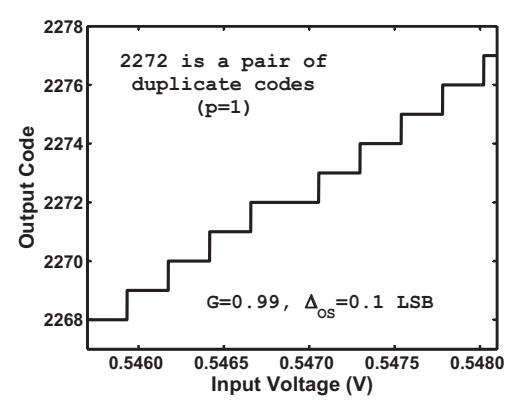

(c)

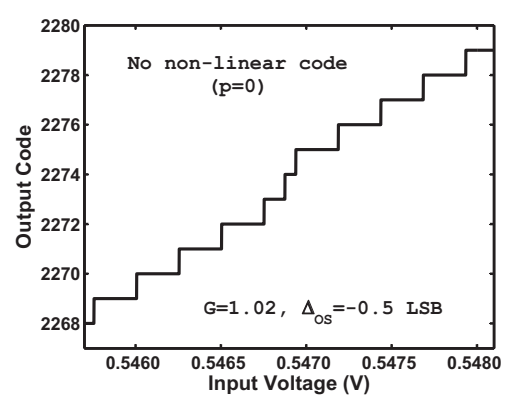

(e)

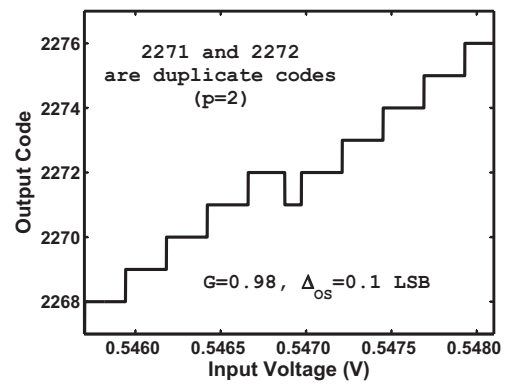

(b)

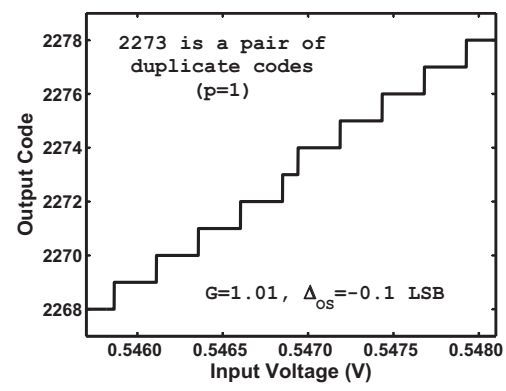

(d)

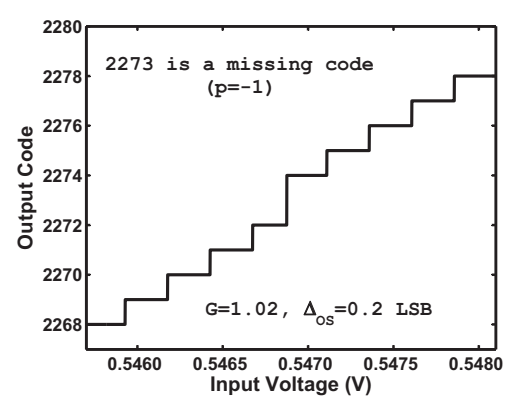

(f)

Figure 4: Typical static characteristics simulated by behavior model for different $G$ and $\Delta_{O S}$. 
Table 2: Theoretical differential non-linearity.

\begin{tabular}{c|c|c|c|c}
\hline \multirow{2}{*}{$p$} & \multicolumn{4}{|c}{ Differential Nonlinearity (DNL) } \\
\cline { 2 - 5 } & TAC & NLC & FAC & LAC \\
\hline$p<0$ & $G-1$ & -1 & $W_{F}-1$ & $W_{L}-1$ \\
\hline$p=0$ & $G-1$ & - & $W_{F}-1$ & $W_{L}-1$ \\
\hline$p=1$ & $G-1$ & \multicolumn{3}{|c}{$W_{F}+W_{L}-1$} \\
\hline \multirow{2}{*}{$p$} & \multirow{2}{*}{ TAC } & & NLC \\
\cline { 3 - 5 } & & TAC+TAC & FAC $+\mathrm{TAC}$ & LAC $+\mathrm{TAC}$ \\
\hline$p=2$ & $G-1$ & - & $W_{F}+G-1$ & $W_{L}+G-1$ \\
\hline$p>2$ & $G-1$ & $2 G-1$ & $W_{F}+G-1$ & $W_{L}+G-1$ \\
\hline
\end{tabular}

characteristic of the SRSS ADC, and is implemented in MATLAB based on the circuit blocks as shown in Fig. 3. The counter generates a digital code increasing from 0 to $2^{M}$ and then from 0 to $2^{N+1}$ for the coarse and fine ramp phases, respectively, which is multiplied with $\Delta_{C}$ and $\Delta_{F}$, respectively, and converted to the ramp voltage $\left(V_{R M P}\right)$ by a DAC. The RIS transfers $V_{R M P}$ to one of the comparator input by taking into account the non-idealities of gain error and offset voltage. By comparing the input voltage $\left(V_{I N}\right)$ and the non-ideal ramp voltage, the comparator decides whether the digital code should be stored into the memory. In the end of one conversion cycle, the final code is obtained by two codes of $m$ and $n$ stored in the memory according to (1).

With $B=12$ bits and $N=6$ bits, the static characteristic of the behavior SRSS ADC is simulated as the function of $\Delta_{O S}$ sweeping from -1LSB to 1LSB, while $G$ is chosen as $0.98,0.99,1.01$, and 1.02 , so as to represent four ranges separated by $\left(1-\frac{1}{2^{N}+1}\right), 1$, and $\left(1+\frac{1}{2^{N}-1}\right)$ according to (5). Fig. 4 shows the typical simulation results, which intuitively exhibits various non-linearity phenomena in the SRSS ADC. From these simulation results, the specifications of non-linearity are extracted, including differential non-linearity (DNL) and the number of NLCs $(p)$ as illustrated as symbols in Fig. 5 ,

With a view to comparison, theoretical values for $p$ and DNL are needed. $p$ 


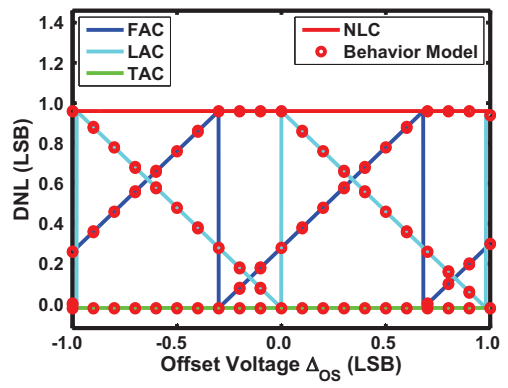

(a)

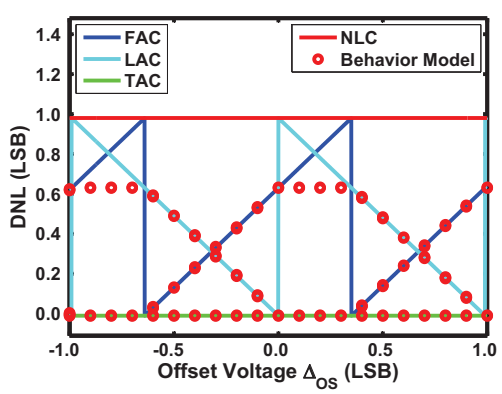

(c)

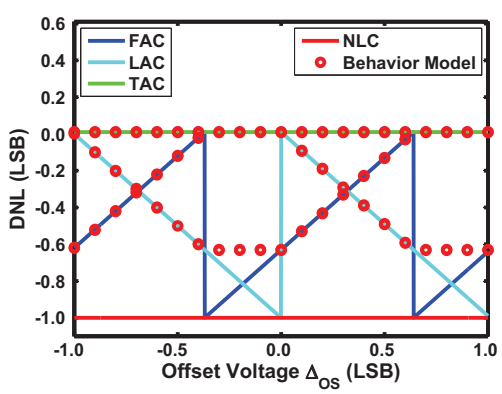

(e)

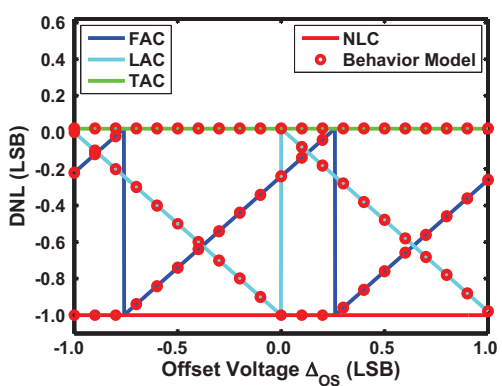

(g)

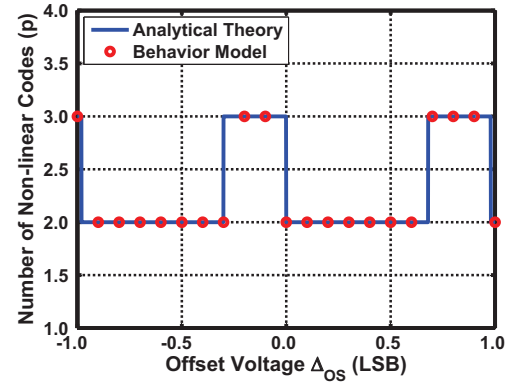

(b)

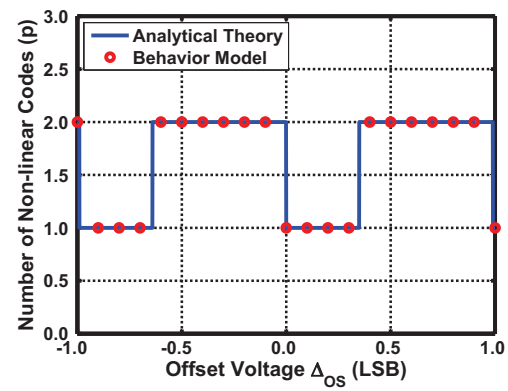

(d)

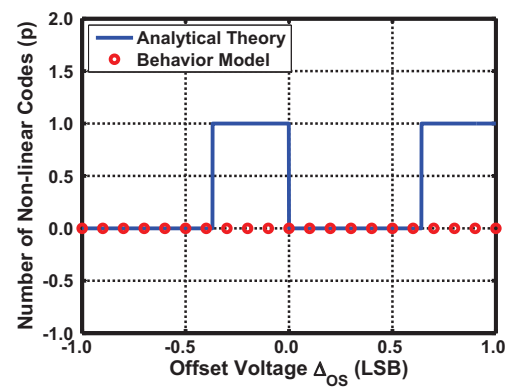

(f)

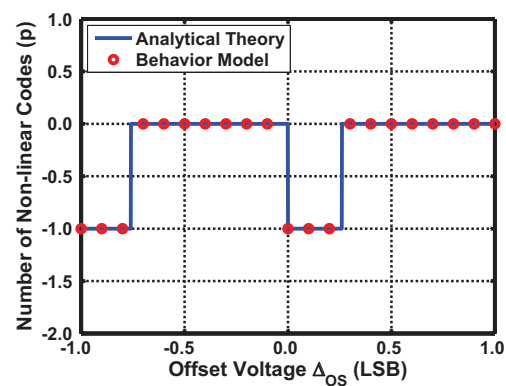

(h)

Figure 5: Comparison of analytical theory (lines) and behavior model (symbols) regarding DNL and number of abnormal codes as the function of offset voltage with ramp gains of (a)(b) 0.98, (c)(d) 0.99, (e)(f) 1.01, and (g)(h) 1.02 . 
has already listed in Table 1 where $p_{0}$ can be calculated by (4). Meanwhile, as listed in Table 2, the theoretical DNLs are derived from the theoretical code widths in Table 1, where $W_{F 0}$ can be calculated by (3) and (4). When $p<0$ and $p>2$, DNLs have four kinds of different values, corresponding to four kinds of codes. When $p$ is in between, the kinds of DNL values are less than four stemming from changes of NLC, FAC, and LAC as follows. When $p=0$, there is no NLC so that the kinds of DNL values are decreased to 3. When $p=1, \mathrm{FAC}$ and LAC in the neighboring fine ramps become a pair of duplicate codes, and merge into one kind of NLC, so that the kinds of DNL values are further decreased to 2 . When $p=2$, FAC and LAC are duplicate with the TACs in the preceding and succeeding fine ramps, respectively, and become two kinds of NLCs, so that the kinds of DNL values are increased to 3.

Fig. 5 compares the theoretical $p$ and DNL (lines) with the behavior simulation results (symbols). For simplicity, the theoretical DNLs in Table. 2 for $p<0$ and $p>2$ are plotted for $G>1$ and $G<1$, respectively, while DNLs for $p=0,1,2$ are discussed individually as below.

- The extracted DNLs and $p$ 's are periodic functions of $\Delta_{O S}$ in that both of them are functions of $r$ in theory while $r$ is a periodic function of $\Delta_{O S}$.

- When $p>2$ or $p<0$, the theoretical values of DNLs and $p$ 's agree with the extracted counterparts as demonstrated in Figs. 蓸(a)|(g) and Figs. 國(b)(h) respectively, for $p=3$ and $p=-1$. Figs. [ [ $[$ (a) (g) also show that the extracted DNLs have four kinds of values as expected in theory, which is manifested in Figs. 4 (a)(f),

- When $p=2$, the theoretical values of DNLs and $p$ 's agree with the ex-

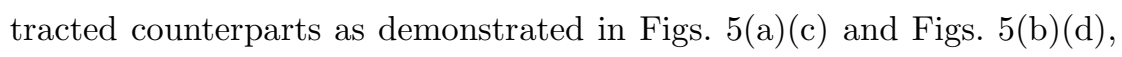
respectively. Figs. 盛(a)(c) also show that the extracted DNLs have three kinds of values as expected in theory which is manifested in Fig. L[(b).

- When $p=1$, Figs. 或 $(\mathrm{c})(\mathrm{e})$ show that the extracted DNLs have two kinds of values as expected in theory, which is manifested in Figs. 4 (c) (d) 
Figs. 國(c)(e) also show that the DNLs of TAC are matched between theory and extraction, while the extracted DNLs of NLC are independent of $\Delta_{O S}$, which is explained as follows. For $G=0.99$ in Fig. [l[ (c) as $r$ increases from 0 , FAC and LAC of the adjacent fine ramps become a pair of duplicate codes and combined as one NLC. Their widths are expressed in Case 2 in Table 1 and therefore, this NLC width is $W_{N 1}=W_{F}+W_{L}=\left(W_{F 0}+\right.$ $r)+\left(W_{L 0}-r\right)=W_{F 0}+G$. Although the same combination also arises for $G=1.01$ in Fig. 國(e) as $r$ decreases from 0 , the difference is that due to $r<0, W_{F}$ and $W_{L}$ are expressed in Case 3 in Table 1, and therefore, this NLC width is $W_{N 2}=W_{F}+W_{L}=\left(W_{F 0}+r\right)+(-r)=W_{F 0}$. However, when extracting $p$ from the behavior model simulation, the algorithm finds a pair of duplicate codes if its code width is greater than $G$. Therefore, due to $W_{N 1}>G$, the former NLC is regarded as a pair of duplicate codes so that the extracted $p$ 's equal 1 in Fig. 徏(d), while due to $W_{N 2} \leqslant G$, the latter NLC is not regarded as a pair of duplicate codes so that the extracted $p$ 's equal 0 rather than 1 in theory in Fig. 或(f)

- When $p=0$, the theoretical values of DNLs and $p$ 's agree with the extracted counterparts as demonstrated in Figs. 國(e)|(g) and Figs. 國(f)|(h) respectively. Figs. [( [e) $(\mathrm{g})$ also show that the extracted DNLs have three kinds of values as expected in theory, which is manifested in Fig. 4(e), for the simulation results of the SRSS ADC behavior model.

\section{Active RIS}

The proposed theory indicates that for the passive RIS in Fig. [1] ta) the linearity constraint imposes a lower bound on $C_{H}$ as

$$
C_{H}>2{ }^{N} C_{P A},
$$

by substituting (2) into (5). Therefore, for a high-resolution SRSS ADC using the passive RIS, $B$ can hardly be equally divided into $M$ and $N$, involving a 


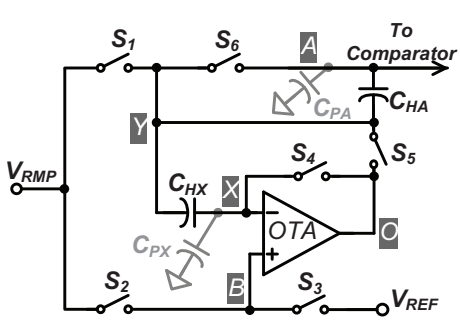

(a)

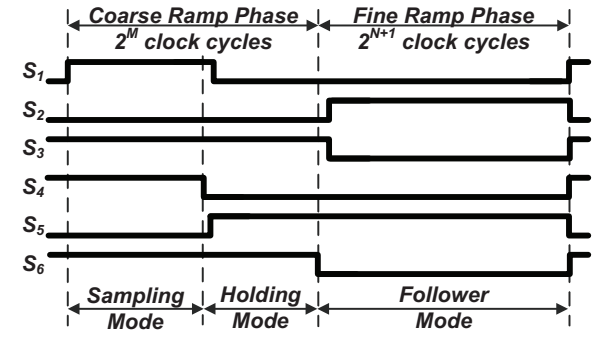

(b)

Figure 6: Proposed active ramp input stage: (a) schematic with $V_{R M P}$ from a ramp generator and $V_{A}$ to a comparator, and (b) timing sequence.

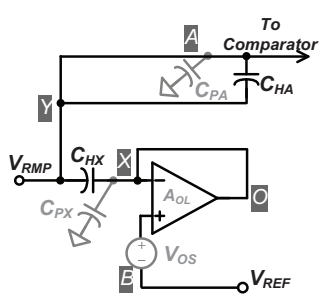

(a)

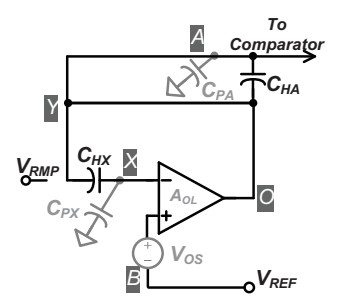

(b)

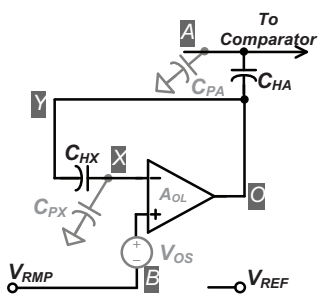

(c)

Figure 7: Equivalent circuits for the active RIS in (a) sampling, (b) holding, and (c) follower modes.

tradeoff between speed, area and power consumption. In order to overcome this drawback, an active RIS is proposed.

\subsection{Operation principle}

Fig. [(a) shows the circuit for the proposed active RIS, which is essentially a flip-around sample-and-hold circuit built by an operational transconductance amplifier (OTA). Controlled by the timing shown in Fig. G(b), the active RIS sequentially operates in three modes as shown in Fig. 7

\subsubsection{Sampling mode}

At the beginning of a coarse ramp phase, $S_{2}$ and $S_{5}$ are turned off, while the others are turned on. The active RIS goes into the sampling mode as shown in 
Fig. [1(a) $V_{R M P}$ from the coarse ramp generator is sampled by the OTA, and simultaneously shorted with $A$ to compare with the input signal $\left(V_{I N}\right)$ by the comparator (not shown). Therefore, the node voltages are

$$
V_{X}=V_{O}=\frac{A_{O L}}{1+A_{O L}}\left(V_{R E F}+V_{O S}\right)
$$

and

$$
V_{Y}=V_{A}=V_{R M P}
$$

where $A_{O L}$ and $V_{O S}$ are the open-loop gain and the offset voltage of the OTA, respectively.

\subsubsection{Holding mode}

Once $V_{R M P}>V_{I N}$, the active RIS goes into the holding mode as shown in Fig. [if (b) in the same way as the flip-around operation [16] as follows. $S_{4}$ turns off first to avoid the signal-dependent charge injection, and then $S_{1}$ and $S_{5}$ change their states immediately. Thus, the instantaneous coarse ramp voltage $\left(V_{H L D}\right)$ is memorized with the node voltages expressed as

$$
V_{A}=V_{Y}=V_{O}=\frac{A_{O L}}{1+A_{O L} \beta_{X}}\left(\beta_{X} V_{H L D}+\frac{V_{R E F}+V_{O S}}{1+A_{O L}}\right),
$$

where

$$
\beta_{X}=\frac{C_{H X}}{C_{H X}+C_{P X}}
$$

\subsubsection{Follower mode}

When the fine ramp phase begins, $S_{6}$ turns off first, which injects signalindependent charges to $A$ due to $V_{A}=V_{Y}$. Then, $S_{2}$ and $S_{3}$ change their states so that Node B is switched from $V_{R E F}$ to $V_{R M P}$, and the active RIS goes

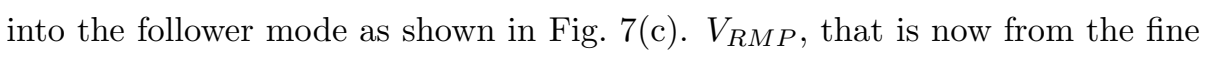
ramp generator, changes the voltage on $B$, and then propagates through $O$ to $A$, whose voltage changes are denoted as $\Delta V_{B}, \Delta V_{O}$ and $\Delta V_{A}$, respectively. Hence, its ramp gain becomes

$$
G=\frac{\Delta V_{A}}{\Delta V_{B}}=\frac{\Delta V_{A}}{\Delta V_{O}} \frac{\Delta V_{O}}{\Delta V_{B}}=\frac{\beta_{A}}{\beta_{X}} \frac{1}{1+\frac{1}{A_{O L} \beta_{X}}}
$$


in that

$$
\frac{\Delta V_{O}}{\Delta V_{B}}=\frac{A_{O L}}{1+A_{O L} \beta_{X}}
$$

and

$$
\frac{\Delta V_{A}}{\Delta V_{O}}=\beta_{A}=\frac{C_{H A}}{C_{H A}+C_{P A}} .
$$

Let $C_{H A}$ and $C_{H X}$ have a nominal value of $C_{H}$, while $C_{P A}$ and $C_{P X}$ have a nominal value of $C_{P}$. In order to achieve this, each of $C_{P A}$ and $C_{P X}$ consists of not only the parasitic capacitance, but also a manually added capacitor that plays a leading role. Assume that $C_{H A}=C_{H}$ and $C_{P A}=C_{P}$, while $C_{H X}$ and $C_{P X}$ deviate from their nominal values by $\Delta C_{H}$ and $\Delta C_{P}$, respectively, i.e., $C_{H X}=C_{H}+\Delta C_{H}$ and $C_{P X}=C_{P}+\Delta C_{P}$. As $A_{O L} \gg 1, \Delta C_{H} \ll C_{H}$, and $\Delta C_{P} \ll C_{P}$, (12) can be rewritten as

$$
G \approx \frac{\beta_{A}}{\beta_{X}}=1+\frac{C_{P}}{C_{H}+C_{P}}\left(\frac{\Delta C_{P}}{C_{P}}-\frac{\Delta C_{H}}{C_{H}}\right)
$$

which depends on the relative deviation denoted as $\frac{\Delta C}{C}=\frac{\Delta C_{P}}{C_{P}}-\frac{\Delta C_{H}}{C_{H}}$.

\subsection{Design constraints}

In order to estimate $C_{H}$ and $C_{P}$, design constraints are discussed. As shown in Fig. [7, the active RIS responds to $V_{R M P}$ in two different feedback configurations with distinct feedback factors $(\beta)$ and capacitive loads $\left(C_{L}\right)$ as expressed by

$$
\beta=1 \text { and } C_{L}=C_{P X}+C_{H X}
$$

in the sampling mode, while

$$
\beta=\beta_{X} \text { and } C_{L}=\frac{C_{P X} C_{H X}}{C_{P X}+C_{H X}}+\frac{C_{P A} C_{H A}}{C_{P A}+C_{H A}}
$$

in the follower mode.

\subsubsection{Linearity constraint}

Substituting (15) into (5), the linearity constraint for the active RIS becomes

$$
C_{H}>\left[\left(2^{N}+1\right)\left|\frac{\Delta C_{P}}{C_{P}}-\frac{\Delta C_{H}}{C_{H}}\right|-1\right] C_{P}
$$


Note that in the active RIS, each of $C_{P X}$ and $C_{P A}$ consists of a manually implemented capacitor besides the parasitic one. Hence, $\Delta C_{P}$ accounts for both mismatch, and the difference in the voltage-dependent input capacitance between the OTA and the comparator.

\subsubsection{Thermal noise constraint}

Regarding the thermal noise [17], the sampled noise power goes directly to $O$ by the flip-around operation, and after combined with the OTA noise power referred to $O$, is delivered to $A$ as an output $\overline{V_{\text {out }}^{2}}$ by a factor of $\beta_{A}^{2}$. For $\overline{v_{\text {out }}^{2}}$ to be less than half of the quantization noise power, the thermal noise constraint is given by

$$
\overline{v_{\text {out }}^{2}}=\left(\frac{k T}{C_{H X}}+\frac{4 k T}{3 \beta_{X} C_{L}}\right) \beta_{A}^{2}<\frac{\Delta_{F}^{2}}{24}
$$

where $k$ is the Boltzmann constant, $T$ is the absolute temperature in degrees Kelvin, and $C_{L}$ is given by (17).

\subsubsection{Power constraint}

For a B-bit ADC, the accuracy is determined by the static and dynamic performance of the OTA. The static deviation must be less than $\Delta_{F} / 2$ for the OTA output at $O$, which imposes a lower limit on the open-loop gain $\left(A_{O L}\right)$ of the OTA given by [18]

$$
A_{O L}>\frac{2^{B+1}}{\beta} .
$$

The dynamic accuracy requires that the exponential settling error at $O$ must be less than $\Delta_{F} / 2$ within a settling time given by a proportion $(n)$ of the clock period $\left(1 / f_{c l k}\right)$, which imposes a lower limit on the gain-bandwidth product (GBW) of the OTA given by [18]

$$
G B W>\frac{(B+1)(\ln 2)}{2 \pi n \beta} \cdot f_{c l k} .
$$

Therefore, the power constraint is written as

$$
I_{i}>\left[(B+1)(\ln 2) \frac{f_{c l k}}{n} \frac{C_{L}}{\beta}\right]\left(\frac{V_{o d}}{2}\right)
$$




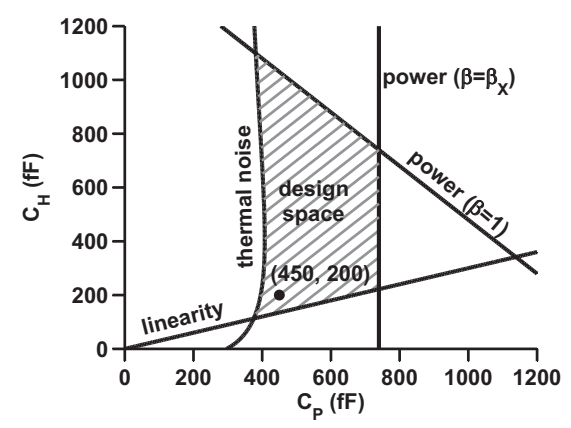

Figure 8: Design space with the selected design point

in that $G B W=\frac{g_{m}}{2 \pi C_{L}}$ and $g_{m}=\frac{2 I_{i}}{V_{o d}}$ [18], where $g_{m}$ is the transconductance of the OTA, $I_{i}$ is the current through the OTA input pair, and $V_{o d}$ is the overdrive voltage. Equ. (22) should be applied to both feedback configurations in (16) and (17).

\subsection{Design space}

The three constraints given by (18), (19), and (22) are embodied by a design space as shown in Fig. 8, after substituting the parameter values listed in Table 3. In Table 3, $V_{o d}$ is simplified for high gain design [18], $\left|\frac{\Delta C}{C}\right|$ is evaluated by simulation, and $T$ is chosen in the cryogenic temperature range for long-wavelength infrared (LWIR) detectors [19]. Therefore, within the design space, $\left(C_{P}, C_{H}\right)=(450 f F, 200 f F)$ is chosen as a design point.

Table 3: Design parameter values for the design space

\begin{tabular}{ccccccccc}
\hline $\begin{array}{c}B \\
(\mathrm{bit})\end{array}$ & $\begin{array}{c}N \\
(\mathrm{bit})\end{array}$ & $\begin{array}{c}V_{F S} \\
(\mathrm{~V})\end{array}$ & $\left|\frac{\Delta C}{C}\right|$ & $\begin{array}{c}T \\
(\mathrm{~K})\end{array}$ & $\begin{array}{c}I_{i} \\
(\mu \mathrm{A})\end{array}$ & $\begin{array}{c}f_{\text {clk }} \\
(\mathrm{MHz})\end{array}$ & $\begin{array}{c}V_{\text {od }} \\
(\mathrm{V})\end{array}$ \\
\hline 12 & 6 & 1 & $2 \%$ & 80 & 100 & 50 & $2 / 3$ & 0.2 \\
\hline
\end{tabular}

\subsection{Simulation and discussion}

With the design point, the active RIS shown in Fig. 6 (a) is designed in a 225 0.18- $\mu \mathrm{m}$ CMOS process with its layout extracted for simulation. Since the other 


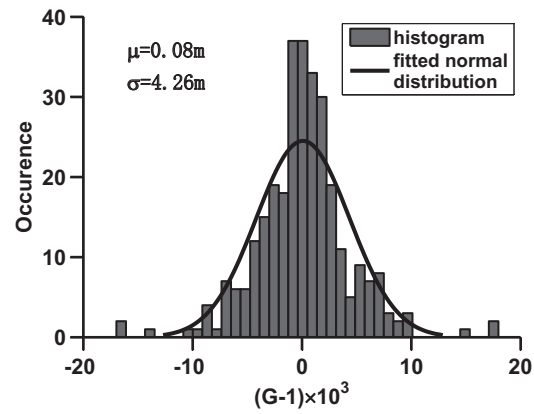

(a)

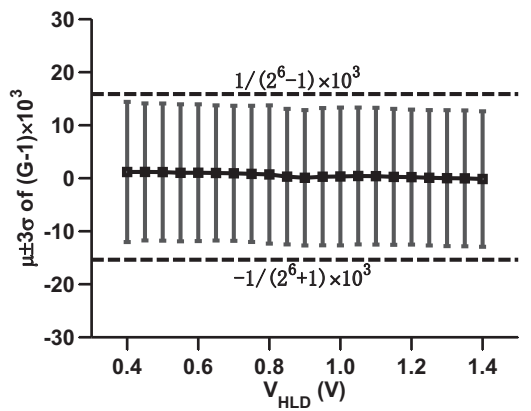

(b)

Figure 9: Monte Carlo simulation results of $G-1$ for active RIS (a) Histogram and fitted normal distribution of 300 samples at $V_{H L D}=0.9 \mathrm{~V}$ (b) $3-\sigma$ error bars as a function of $V_{H L D}$

blocks of the SRSS ADC in Fig. 3 are also necessary to perform simulation, the ramp generator, timing control, and comparator are implemented by veriloga, verilog, and circuit, respectively. The comparator employs a preamplifier with a tail current to weaken the dependence of its input capacitance on $V_{A}$ [20].

The simulation is carried out at $80 \mathrm{~K}$, although it is beyond the valid range of the device models provided by the foundry. Due to the absence of accurate models [21], enough margins have to be left for the increases of channel conductance and threshold voltages [22].

As the mismatch plays an important role in $G$, Monte Carlo mismatch simulation is performed for the active RIS. Afterwards, the ramp gain error $\Delta G=G-1$ is extracted and statistically analyzed. Fig. G(a) shows the histogram of 300 samples at $V_{H L D}=0.9 \mathrm{~V}$, to which a normal distribution is fitted with the mean value $(\mu)$ and standard deviation $(\sigma)$ obtained. In this way, the statistical properties of $\Delta G$ for different $V_{H L D}$ 's are obtained and depicted as 3 - $\sigma$ error bars as shown in Fig. depends on $V_{H L D}$, and $\Delta G$ satisfies (5) even with 3- $\sigma$ deviations.

The 12-bit SRSS ADC with the active RIS is simulated with its output codes 


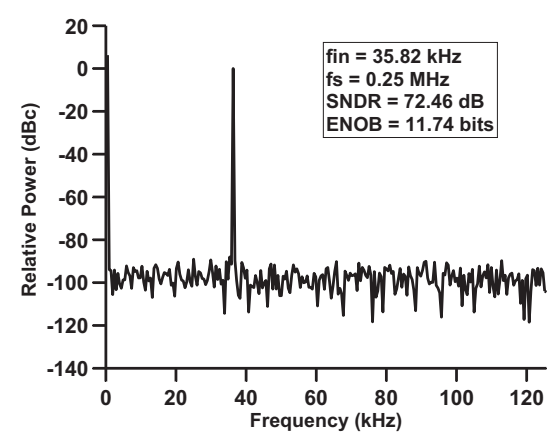

Figure 10: Simulation result of 512-point FFT spectrum for a $35.82-\mathrm{kHz}$ input signal at $0.25 \mathrm{MS} / \mathrm{s}$.

analyzed by FFT as shown in Fig. 10, where the sampling rate approximately equals $f_{c l k} /\left(2^{M}+2^{N+1}\right)$.

The total capacitance is $1.3 p F$ in this design. While for the passive RIS in Fig. [1] if $C_{P A}=50 f F, C_{H}>3.2 p F$ according to (7), to which over $20 \%$ margin should be added for process variations. Taking $C_{H}=4 p F$ in the passive RIS for example, the capacitor area can be reduced by $1350 \mu \mathrm{m}^{2}$ using $2 \mathrm{fF} / \mu \mathrm{m}^{2}$ MetalInsulator-Metal (MIM) capacitors, which is partly cancelled out by the OTA area about $480 \mu \mathrm{m}^{2}$. The OTA power consumption is simulated about $180 \mu \mathrm{W}$, which seems to be a penalty from a single ADC point of view. However, in a column-parallel architecture, it is expected to be cancelled out by the power reduction of the global buffers that drive $V_{R M P}$ and $V_{R E F}$ due to the reduced capacitive loads.

255 4. Conclusions

This paper proposed a theory quantifying the ramp gain error effect of RIS in SRSS ADCs, which is strongly supported by the behavior model simulation. The active RIS is proposed for SRSS ADCs, and designed under the guidance of the design space. Circuit simulation results verify its enhancement on linearity. It is a potential solution to the parasitic capacitance problem of RIS. 


\section{Acknowledgments}

This paper supported by the State Key Program of National Natural Science of China (Grant No. 61234002), the National Natural Science Foundation of China (Grant No. 61574041), the National Science Foundation for Distinguished Young Scholars of China (Grant No. 61525401), and by Key Laboratory of Infrared Imaging Materials and Detectors, Shanghai Institute of Technical Physics, Chinese Academy of Sciences.

\section{References}

[1] W. Yang, O.-B. Kwon, J.-I. Lee, G.-T. Hwang, S.-J. Lee, An integrated 800x600 CMOS imaging system, in: IEEE ISSCC Dig. Tech. Papers, 1999, pp. $304-305$.

[2] T. Sugiki, S. Ohsawa, H. Miura, M. Sasaki, N. Nakamura, I. Inoue, M. Hoshino, Y. Tomizawa, T. Arakawa, A $60 \mathrm{~mW} 10$ b CMOS image sensor with column-to-column FPN reduction, in: IEEE ISSCC Dig. Tech. Papers, 2000, pp. 108-109.

[3] K. Findlater, R. Henderson, D. Baxter, J. Hurwitz, L. Grant, Y. Cazaux, F. Roy, D. Herault, Y. Marcellier, SXGA pinned photodiode CMOS image sensor in 0.35 um technology, in: IEEE ISSCC Dig. Tech. Papers, 2003, pp. $218-489$.

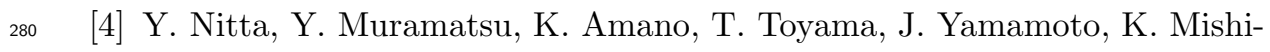
na, A. Suzuki, T. Taura, A. Kato, M. Kikuchi, Y. Yasui, H. Nomura, N. Fukushima, High-speed digital double sampling with analog CDS on column parallel ADC architecture for low-noise active pixel sensor, in: IEEE ISSCC Dig. Tech. Papers, 2006, pp. 2024-2031.

${ }_{285}^{25}$ [5] T. Takayanagi, M. Shirakawa, K. Mitani, M. Sugawara, S. Iversen, J. Moholt, J. Nakamura, E. R. Fossum, A 1.25-inch 60 frames/s 8.3-Mpixel digital output CMOS image sensor, IEEE Journal of Solid-State Circuits 40 (11) (2005) 2305-2314. 
[6] S. Matsuo, T. J. Bales, M. Shoda, S. Osawa, K. Kawamura, A. Andersson, M. Haque, H. Honda, B. Almond, Y. Mo, J. Gleason, T. Chow, I. Takayanagi, A very low column FPN and row temporal noise 8.9 Mpixel, 60 fps CMOS image sensor with 14 bit column parallel SA-ADC, IEEE Trans. Electron. Device 56 (11) (2009) 2380-2389.

[7] M. Liu, Y. Xie, Z. Zhu, A 67.2 dB SNDR 1.8-V 12-bit 2-MS/s SAR AD$\mathrm{C}$ without calibration, Analog Integrated Circuits and Signal Processing 86 (1) (2016) 151-158.

[8] W. Guo, S. Liu, Z. Zhu, An asynchronous 12-bit 50MS/s rail-to-rail pipeline-SAR ADC in 0.18 $\mu \mathrm{m}$ CMOS, Microelectronics Journal 52 (2016) $23-30$.

[9] Z. Zhu, Z. Qiu, M. Liu, R. Ding, A 6-to-10-bit 0.5V-to-0.9V reconfigurable 2MS/s power scalable SAR ADC in $0.18 \mu \mathrm{m}$ CMOS, IEEE Trans. Circuits and Systems I: Regular Papers 62 (3) (2015) 689-696.

[10] Y. Chae, J. Cheon, S. Lim, M. Kwon, K. Yoo, W. Jung, D.-H. Lee, S. Ham, G. Han, A 2.1 M pixels, 120 frame/s CMOS image sensor with columnparallel ADC architecture, IEEE Journal of Solid-State Circuits 46 (1) (2011) 236-247.

[11] M.-W. Seo, S.-H. Suh, T. Iida, T. Takasawa, K. Isobe, T. Watanabe, S. Itoh, K. Yasutomi, S. Kawahito, A low-noise high intrascene dynamic range CMOS image sensor with a 13 to $19 \mathrm{~b}$ variable-resolution column-parallel folding-integration/cyclic ADC, IEEE Journal of Solid-State Circuits 47 (1) (2012) 272-283.

[12] M.-W. Seo, T. Sawamoto, T. Akahori, Z. Liu, T. Iida, T. Takasawa, T. Kosugi, T. Watanabe, K. Isobe, S. Kawahito, A low-noise high-dynamic-range 17-b 1.3-megapixel 30-fps CMOS image sensor with column-parallel twostage folding-integration/cyclic ADC, IEEE Trans. Electron. Device 59 (12) (2012) 3396-3400. 
[13] M. F. Snoeij, A. J. P. Theuwissen, K. A. A. Makinwa, J. H. Huijsing, Multiple-ramp column-parallel ADC architectures for CMOS image sensors, IEEE Journal of Solid-State Circuits 42 (12) (2007) 2968-2977.

[14] S. Lim, J. Lee, D. Kim, G. Han, A high-speed CMOS image sensor with column-parallel two-step single-slope ADCs, IEEE Trans. Electron. Device 56 (3) (2009) 393-398.

[15] J. Lee, H. Park, B. Song, K. Kim, J. Eom, K. Kim, J. Burm, High framerate VGA CMOS image sensor using non-memory capacitor two-step singleslope ADCs, IEEE Trans. Circuits and Systems I: Regular Papers 62 (9) (2015) 2147-2155.

[16] B. Razavi, Design of Analog CMOS Integrated Circuits, McGraw-Hill, 2001, Ch. 12, pp. 423-439.

[17] R. Schreier, J. Silva, J. Steensgaard, G. Temes, Design-oriented estimation of thermal noise in switched-capacitor circuits, IEEE Trans. Circuits and Systems I: Regular Papers 52 (11) (2005) 2358-2368.

[18] W. Sansen, Analog Design Essentials, Springer, 2006.

[19] Handbook of Optics, Third Edition, McGraw-Hill, 2010.

[20] Y.-Z. Lin, C.-C. Liu, G.-Y. Huang, Y.-T. Shyu, Y.-T. Liu, S.-J. Chang, A 9-bit 150-MS/s subrange ADC based on SAR architecture in 90-nm CMOS, IEEE Trans. Circuits and Systems I: Regular Papers 60 (3) (2013) 570-581.

[21] A. Akturk, M. Holloway, S. Potbhare, D. Gundlach, B. Li, N. Goldsman, M. Peckerar, K. Cheung, Compact and distributed modeling of cryogenic bulk MOSFET operation, IEEE Trans. Electron. Device 57 (6) (2010) 1334-1342.

[22] S. Hanamura, M. Aoki, T. Masuhara, O. Minato, Y. Sakai, T. Hayashida, Operation of bulk CMOS devices at very low temperatures, IEEE Journal of Solid-State Circuits 21 (3) (1986) 484-490. 